\title{
Modelo de Mensuração de Sequenciamentos de Objetos de Aprendizagem
}

\author{
Lázaro De Almeida ${ }^{1}$, Robinson Vida Noronha ${ }^{1}$ \\ ${ }^{1}$ Departamento Acadêmico de Informática (DAINF) - Universidade Tecnológica \\ Federal do Paraná (UTFPR) \\ Av. 7 de setembro 3165 - 80230-901 Curitiba - PR - Brasil \\ lazaro6almeida@gmail.com, vidadutfpr.edu.br
}

\begin{abstract}
How to measure a sequence or order of learning objects in order to determine whether such sequencing is suitable to instructional goals an educator to achieve? Based on this question this paper proposes a computational model for measuring the sequencing of learning objects based on the concept of Assimilation Costs - ASSIMILATE. The objective of this model is being able to assist the educator with tasks of planning, implementation and monitoring of instructional process, besides serving as a support for the development of a validation system for sequences of learning objects.
\end{abstract}

Resumo: Como mensurar uma sequência ou ordenamento de objetos de aprendizagem com a finalidade de determinar se tal sequenciamento é adequado aos objetivos instrucionais que um educador deseja alcançar? Com base nessa questão este artigo propõe um modelo computacional para mensuração do sequenciamento de objetos de aprendizagem baseado no conceito de custos de assimilação - ASSIMILATE. O objetivo deste modelo é poder auxiliar o educador com as tarefas de planejamento, execução e acompanhamento do processo instrucional, além de poder servir de suporte para o desenvolvimento de um sistema de validação de sequências de objetos de aprendizagem.

\section{Introdução e Desafios Abordados}

Um dos problemas que pode ser encontrado por educadores e autores de materiais instrucionais é como definir a sequência de acesso do aprendiz aos objetos de aprendizagem. [Phillips and Kane 1973] asseveram que existe evidência substancial que o processo de aprendizagem se dê de maneira hierárquica dos níveis mais elementares de conhecimento aos mais elaborados.

De maneira similar, Robert Gagne apresentou que o planejamento instrucional é basicamente um problema de definição de sequências de assuntos, de definição de tarefas e subtarefas [Gagne 1963]. Além disso, a determinação da ordem hierárquica de apresentação e execução das subtarefas de aprendizagem que vão das simples as mais complexas continua sendo um problema relevante uma vez que algumas perguntas continuam sem respostas [Gagne 1968]:

- Como determinar se uma hierarquia de aprendizagem é adequada?

- Uma hierarquia de aprendizagem representa um caminho único para uma tarefa final ou representa o caminho mais eficiente de aprendizagem? 
Logo, pode-se considerar que uma hierarquia não poderia representar apenas um caminho único ou mais eficiente para determinado aprendiz. De maneira geral, uma hierarquia poderia representar a expectativa provável mais positiva para uma amostra de aprendizes [Gagne 1968].

Apesar das evidências encontradas em outros estudos sobre a importância das hierarquias de aprendizagem [Gagne 1962, 1963, 1968; Gagne and Paradise 1961; Miller and Phillips 1974; Phillips and Kane 1973; Resnick 1975; Trembath and White 1975] não há um método ou uma forma de mensurar um sequenciamento de atividades instrucionais como os objetos de aprendizagem.

Tradicionalmente, a práxis do educador é o principal parâmetro para a validação de sequências de objetos de aprendizagem. Enquanto isso, a abordagem de validação baseada em tentativa e erro se mostra tediosa e bastante custosa, embora sua validade não seja questionada [Phillips and Kane 1973].

Com base nas duas questões levantadas anteriormente em [Gagne 1968] e a necessidade de validação de sequências de objetos de aprendizagem menos custosas que a tentativa e erro depreende-se a necessidade de mecanismos de mensuração do sequenciamento de objetos de aprendizagem para que seja possível uma validação objetiva desse sequenciamento.

Para tentar preencher essa lacuna, este artigo descreve um modelo de mensuração do sequenciamento de objetos de aprendizagem. Essa mensuração é feita por uma métrica multidimensional, definida pelo grupo de pesquisa de Informática Aplicada em Educação da UTFPR, chamada de Custos de Assimilação.

A finalidade deste modelo é tentar auxiliar o educador nas tarefas de planejar, executar e acompanhar o processo instrucional. Além disso, espera-se poder fornecer suporte para o desenvolvimento de um sistema de validação de sequenciamento de objetos de aprendizagem, em trabalhos futuros, a partir dos objetivos instrucionais que o educador determinar.

Para descrever esse modelo computacional e os Custos de Assimilação, este artigo está estruturado em duas partes: a primeira refere-se ao Estado da Arte, apresentando os conceitos e sistemas que tratam dos sequenciamentos de objetos de aprendizagem; a segunda parte desenvolve o modelo descrevendo seus elementos estruturais e os Custos de Assimilação.

\section{Estado da Arte}

Objetos de aprendizagem são elementos instrucionais, baseados no paradigma de orientação a objetos da Ciência da Computação, e que podem ser reutilizados em diferentes contextos [Wiley 2006]. A reusabilidade faz com que esses objetos possam ser montados de diferentes maneiras conforme os objetivos instrucionais que se deseje alcançar [Reigeluth 1999].

No contexto da instrução computadorizada, para que seja possível a reutilização e composição automatizada de objetos de aprendizagem é necessário que o sistema disponha de informações sobre o planejamento instrucional. Essas informações darão fundamento para as decisões de sequenciamento dos objetos de aprendizagem e serão determinantes para o sucesso instrucional desses sistemas. [Wiley 2006]. 
Em [Murray 1999] os sistemas autorais no sequenciamento de curso e do currículo são aqueles que organizam as unidades instrucionais ou elementos do currículo em uma hierarquia de cursos, módulos, lições, apresentações que são relacionados como pré-requisitos, partes e outros relacionamentos. As unidades instrucionais tipicamente possuem objetivos instrucionais. Alguns dos sistemas analisados por Murray podem incluir unidades instrucionais que fazem o tratamento de erros ou possuem material para remediação desses erros.

Nessa perspectiva [Murray 1999], esses sistemas são vistos como ferramentas para auxiliar os educadores na criação do planejamento instrucional. O sequenciamento inteligente das unidades instrucionais (conteúdo, ou tópicos) é o centro destes sistemas. $\mathrm{Na}$ perspectiva do estudante, estes sistemas podem parecer idênticos aos demais sistemas de aprendizagem computadorizados. Contudo, a diferença está no sequenciamento do conteúdo que é determinado dinamicamente baseado no desempenho do estudante, nos objetivos da lição e entre os relacionamentos dos módulos do curso.

Além disso, o sequenciamento de cursos é uma tecnologia bem estabelecida no campo dos Sistemas Tutores Inteligentes - STI [Brusilovsky and Vassileva 2003]. Sendo que essa tecnologia tem como objetivo o sequenciamento de cursos e a geração de cursos individualizados que possam levar o estudante mais próximo do objetivo final da instrução.

Um dos trabalhos na área de sequenciamento e planejamento de currículo foi o de [Peachey and McCalla 1986]. Esse trabalho aplicou e estendeu algumas das técnicas de inteligência artificial de planejamento e controle de ações para robôs para uma arquitetura de instrução auxiliada por computador.

Alguns sistemas mais recentes de STI tem buscado se apoiar em ontologias para a geração de sequências com os apresentados em [Chi 2009; Kontopoulos et al. 2008; Vidal-Castro et al. 2012]. Nesses sistemas o objetivo é a construção automatizada de sequenciamento de unidades educacionais autônomas ou objetos de aprendizagem utilizado. Nessa tarefa, algumas técnicas foram investigadas, tais como: ontologias de habilidades ou competências [Kontopoulos et al. 2008]; regras semânticas para determinação do sequenciamento [Chi 2009; Vidal-Castro et al. 2012] de modo a tentar se ajustar a cada estudante. Contudo, nenhum desses trabalhos apresenta uma forma de mensurar o sequenciamento gerado.

Outro tipo de sistemas instrucionais são os Sistemas de Hipermídia Adaptativa (SHA). Esses são um avanço nos sistemas de hipermídia que eram focados apenas na apresentação de conteúdos instrucionais dispostos no hiperespaço por meio de hiperlinks. Nesses sistemas é construído um modelo dos objetivos, preferências e conhecimento de cada usuário com a finalidade de se adaptar às necessidades daquele usuário [Brusilovsky 2001].

Nesses sistemas existe um modelo explícito do conhecimento do domínio que pode ser ensinado conhecido como espaço do domínio. O espaço do domínio é formado por um conjunto de pequenos elementos de conhecimento que podem ser nominados de diferentes formas conforme o sistema instrucional: conceitos, itens, tópicos, elementos de conhecimento, objetivos de aprendizagem, produtos de aprendizagem. Eles são os fragmentos elementares do espaço de conhecimento [Brusilovsky 2001]. 
Outro modelo de sequenciamento utilizado em SHA é o apresentado por [Zuasnábar et al. 1999]. Nesse modelo, utiliza-se roteiros para definir a sequência das atividades. Esse modelo prevê mecanismos de auxílio ao estudante na tarefa de seguir um roteiro definido pelos professores. A vantagem dessa abordagem está na possível redução da desorientação e da sobrecarga cognitiva, uma vez que os estudantes seguiriam roteiros predefinidos, sendo reduzida a necessidade de tomada de decisões sobre qual material estudar. Além disso, essas sequências de informações definidas facilitariam alcançar os objetivos previstos pelo professor ao desenvolver o seu curso.

Pode-se observar, na revisão da literatura realizada, que nenhum desses trabalhos propõe uma forma de mensurar a sequencia dos objetos de aprendizagem apresentados ao estudante. Acredita-se que uma forma de mensuração possa vir a ser útil como elemento de comparação e validação de diferentes sequências de objetos de aprendizagem que possuam um mesmo objetivo instrucional.

\section{Modelo de Mensuração de Sequenciamento de Objetos de Aprendizagem}

A Figura 1, a seguir, apresenta o modelo computacional investigado por esta pesquisa. Os seus elementos serão explicitados nas seções seguintes: 3.1 Elementos Estruturais e 3.2 Custos de Assimilação (CA).

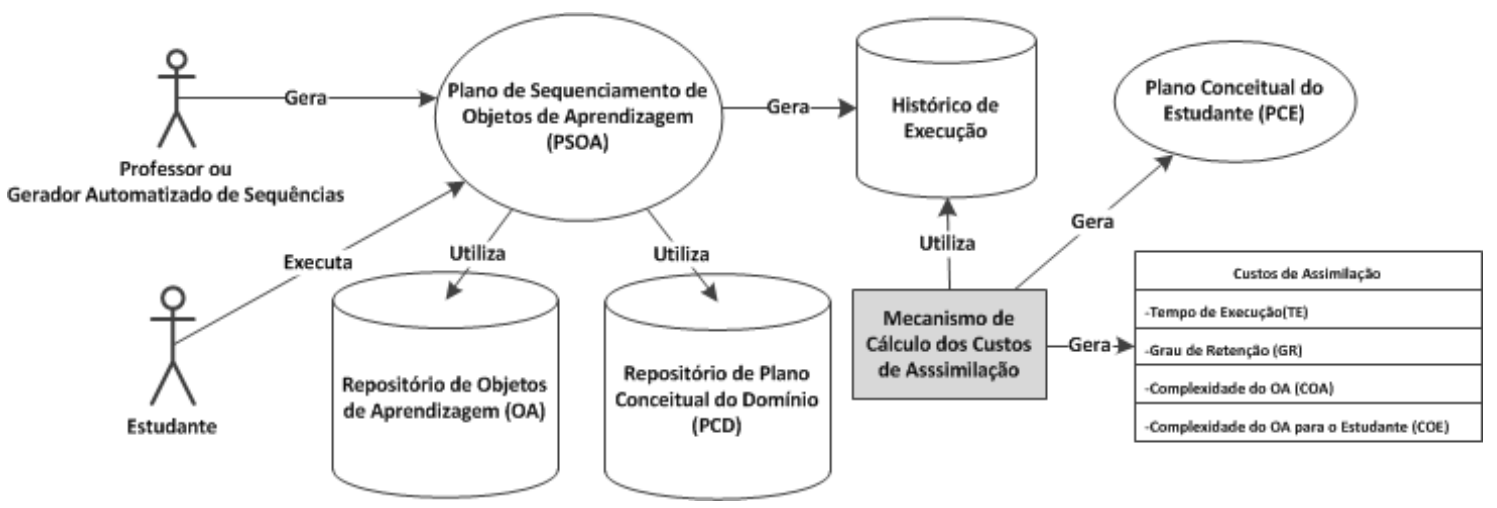

Figura 1 - ASSIMILATE: Modelo de Mensuração de Sequenciamento de Objetos de Aprendizagem

\subsection{Elementos Estruturais}

Os elementos estruturais que o ASSIMILATE irá utilizar são: Plano Conceitual do Domínio (PCD), Plano Conceitual do Estudante (PCE) e o Plano de Sequenciamento dos Objetos de Aprendizagem (PSOA). Esses elementos estruturais serão apresentados nas Seções 3.1.1 a 3.1.3

\subsubsection{Plano Conceitual do Domínio (PCD)}

O plano conceitual do domínio (PCD) é uma forma de representação hierárquica do conhecimento sobre determinado domínio. Esse plano foi baseado nos trabalhos de [Brusilovsky 2001, 1992; Brusilovsky and Vassileva 2003; Gordon 2000; Karampiperis and Sampson 2004, 2004; Murray 1999; Peachey and McCalla 1986; Sicilia et al. 2011; Vidal-Castro et al. 2012] que apresentaram diferentes formas de representar conhecimentos dentro de um sistema instrucional.

O PCD utiliza uma estrutura de grafo acíclico dirigido para representar a dependência entre os conceitos de um domínio. A Figura 2 apresenta um exemplo 
abstrato da representação de um PCD. Nessa figura, a seta indica a dependência de conceitos. Por exemplo, nessa figura, $\mathrm{C} 1$ é dependente de $\mathrm{C} 2$ que por sua vez é dependente de $\mathrm{C} 3$ e $\mathrm{C} 4$ e assim por diante.

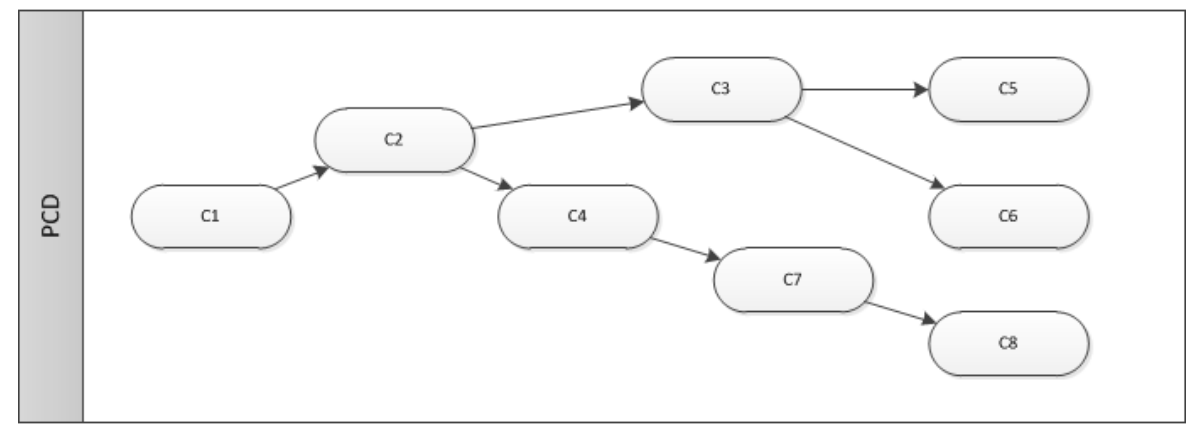

Figura 2 - Plano Conceitual de Domínio (PCD)

Os conceitos considerados mais complexos são aqueles que apresentam o maior número de conceitos dependentes. Na Figura 2 o conceito mais complexo é o $\mathrm{C} 1$ por possuir o maior número de dependências enquanto os conceitos mais simples ou elementares do domínio são os conceitos C5, C6 e C8.

\subsubsection{Plano Conceitual do Estudante (PCE)}

Nos STI a representação dinâmica do conhecimento e habilidades emergentes do estudante, conforme definição de [Nwana 1990] é chamada de modelo do estudante. A função do Plano Conceitual do Estudante (PCE) pode ser considerada a mesma do modelo do estudante nos STI. Optou-se por essa terminologia para enfatizar a dimensão do estudante enquanto um dos planos necessários para a mensuração e também o elemento conceito dentro do modelo.

O PCE é construído utilizando o histórico da execução do sequenciamento dos OA pelo estudante. Os seus valores são determinados a partir do valor do Grau de Retenção de cada OA. A determinação do grau de retenção será apresentada na sessão 3.2.1.2 Grau de Retenção (GR).

O PCE no modelo proposto por este trabalho será representado por um espaço de conceitos autônomos, não dependentes, não ordenados e não relacionados.

\subsubsection{Plano de Sequenciamento dos Objetos de Aprendizagem (PSOA)}

O Plano de Sequenciamento de Objetos de Aprendizagem - PSOA, do mesmo modo como o PCD, é representado por meio de uma estrutura de grafos acíclicos dirigidos onde a seta indica o fluxo com que os Objetos de Aprendizagem (OA) devem ser executados. Um pequeno exemplo abstrato é apresentado na Figura 3.

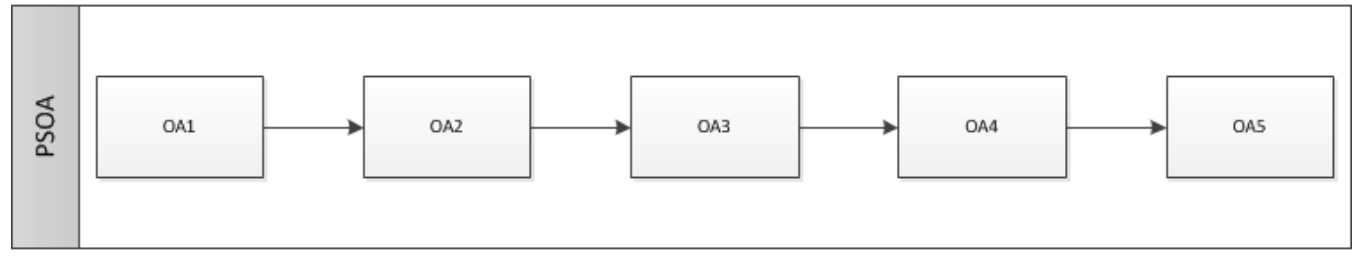

Figura 3 - Plano de Sequenciamento de Objetos de aprendizagem (PSOA) 
A Figura 3 exemplifica uma sequência de objetos de aprendizagem conforme uma possível ordem de execução. Nessa figura um hipotético estudante teria executado a sequência de objetos de aprendizagem: OA1, OA2, OA3, OA4 e OA5 nesta ordem.

Existe um relacionamento entre o PCD e o PSOA. Cada OA corresponde a um ou mais conceitos do PCD, conforme ilustrado na Figura 4. Nessa figura, uma seta indica o relacionamento entre os elementos dos planos PSOA e PCD. Por exemplo, o OA1 poderia estar relacionado com o $\mathrm{C} 7$, o $\mathrm{OA} 3$, por sua vez, com o $\mathrm{C} 5$ e $\mathrm{C} 4$, conforme exemplifica a Figura 4. Esses relacionamentos entre os elementos contidos nos planos PCD e PSOA será empregado para determinar o Custo de Assimilação.

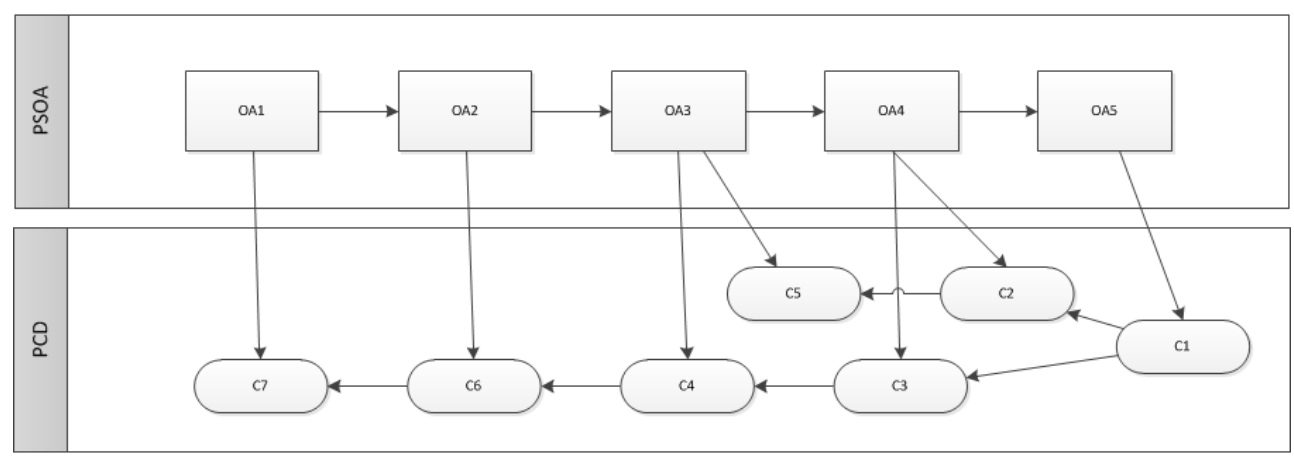

Figura 4 - Relacionamento entre PCD e PSOA

\subsection{Custos de Assimilação (CA)}

Assimilação é um conceito proveniente dos trabalhos do psicólogo suíço Jean Piaget [Piaget 1979]. Assimilação é a integração de qualquer espécie de realidade em uma estrutura. De acordo com [Piaget 1979], a assimilação que é fundamental na aprendizagem, e é a relação fundamental do ponto de vista das aplicações pedagógicas ou didáticas.

Baseado nesse conceito, o processo de assimilação é definido como sendo a demonstração comportamental do conteúdo do objeto de aprendizagem. Por exemplo, o aprendiz teve contato com o conteúdo do objeto de aprendizagem e é capaz de demonstrar novas habilidades por meio de uma verificação como um teste.

Desse modo, define-se aqui o conceito de Custos de Assimilação (CA) como o esforço necessário para que o estudante seja capaz de assimilar determinado objeto de aprendizagem.

Essa transposição de conceitos teóricos para modelos computacionais não é novidade e já foi apresentada em diversos trabalhos que podem ser encontrados na literatura. Como por exemplo, [Murray and Arroyo 2002] e [Katuk and Ryu 2010]. No primeiro exemplo, houve a transposição do conceito de Zona de Desenvolvimento Proximal de Vygotsky para um conceito operacional aplicado a um sistema adaptativo instrucional. O trabalho [Katuk and Ryu 2010] operacionalizou o conceito de Experiência de Fluxo (Flow), do psicólogo húngaro Csikszentmihalyi, como a relação ótima entre habilidade do aprendiz e o nível de desafio dos objetos de aprendizagem executados para encontrar caminhos ótimos de aprendizagem em sequenciamentos de currículos dinâmicos. 


\subsubsection{Variáveis constituintes}

Tendo sido apresentados os elementos estruturais, as seções 3.2.1.1 a 3.2.1.4 descreverão as variáveis que irão constituir os Custos de Assimilação (CA): Tempo de Execução (TE), Grau de Retenção (GR), Complexidade do Objeto de Aprendizagem (COA) e Complexidade do Objeto de Aprendizagem para o Estudante (COE).

\subsubsection{Tempo de Execução (TE)}

O tempo de execução (TE) é definido como o tempo necessário para que um estudante execute um OA dentro de um SOA e pode ser representado por qualquer unidade de tempo. Essa variável é função da ordem ou sequência de execução dos Objetos de Aprendizagem. Acredita-se que uma alteração nessa sequência possa alterar o valor de seu tempo de execução para o estudante.

Um OA mais complexo apresentado antes de um mais simples poderia levar mais tempo ou mesmo chegar a não ser realizado por causa da alteração de ordem. Do mesmo modo, se um OA mais simples fosse posicionado após um OA mais complexo o TE da atividade provavelmente seria menor do que se fosse posicionada antes.

Essa variável é obtida subtraindo-se o tempo inicial da execução de um OA do tempo final de execução de um OA.

\subsubsection{Grau de Retenção (GR)}

O grau de retenção indica a porcentagem do conteúdo do OA que o estudante conseguiu reter como parte de seu conhecimento.

Essa variável é obtida por meio de um pós-teste ao OA em que o valor deve estar entre 1 (inteiro domínio do conteúdo) e 0 (nenhum domínio do conteúdo). Podendo ser dado pela fórmula:

$$
G R=(\text { quantidade de acertos }) /(\text { quantidade de itens })
$$

\subsubsection{Complexidade do Objeto de Aprendizagem (COA)}

A complexidade do objeto de aprendizagem (COA) é a relação que um OA possui com os conceitos que o integram e que estão representados em um PCD.

Para obtenção da COA de um PCD. Tem-se que a COA é o somatório da complexidade dos conceitos que integram aquele $\mathrm{OA}$ :

$$
O E=\sum c_{\text {conceito }}
$$

Onde:

$$
C_{\text {conceito }}=1+n^{\circ} \text { de conceitos dependentes }
$$

Exemplo: Na Figura 5 o OA5 possui $\mathrm{COE}$ de 7 , pois compreende o somatório de conceitos que no exemplo é apenas $\mathrm{C} 7$ cuja complexidade é $1+6\left(\mathrm{n}^{\mathrm{o}}\right.$ de conceitos dependentes).

\subsubsection{Complexidade do Objeto de Aprendizagem para o estudante (COE)}

A complexidade do OA para o estudante é a relação entre a Complexidade do Objeto de Aprendizagem - COA e o conhecimento prévio do estudante representado por meio do seu PCE. Isso serve para indicar que a complexidade de um OA tende a zero caso o estudante possua os conceitos em seu PCE em nível avançado. 
Essa variável é obtida antes da execução do OA. Ela é obtida pela fórmula:

$$
C O E=C O A-\sum_{i}^{n} G R
$$

Onde:

$\sum_{i}^{n} G R$ : somatório dos graus de retenção que o estudante obteve e que estão vinculados aquele $\mathrm{OA}$.

Exemplo: um estudante executando o SOA da Figura 5 obteve GR de 0,7 na OA1. Este estudante vai realizar o OA2 que possui COA de valor 2. Como este estudante obteve GR de 0,7 na OA1 isso determina que o mesmo possua o valor de 0,7 para $\mathrm{C} 7$. Como o estudante não tem conhecimento de C6 então o $\mathrm{COE}$ será de $2-0,7=1,3$.

\subsubsection{Representação dos Custos de Assimilação (CA)}

A partir da apresentação das variáveis constituintes do CA, optou-se por representar os CA por um conjunto de tuplas $(a)$.

$$
C A=\left(a_{1}, a_{2}, \ldots a_{n}\right)
$$

Onde cada tupla $a$ é representada pelo valor de referência para cada OA.

$$
a=\left\{i d E, T E_{O A_{1}}, G R_{O A_{1}}, C O E_{O A_{1}}, C O A_{O A_{1}}, \ldots, T E_{O A_{n}}, G R_{O A_{n}}, C O E_{O A_{n}}, C O A_{O A_{n}}\right\}
$$

Onde:

$i d E=$ identificador único do estudante

\section{Considerações Finais e Trabalhos Futuros}

O presente trabalho propôs um modelo computacional de mensuração de sequenciamento de objetos de aprendizagem baseado em Custos de Assimilação. Acredita-se que tal modelo possa servir para:

- Auxiliar os educadores nas tarefas de planejamento, execução e acompanhamento do processo instrucional.

- Investigação científica e coleta de informações referentes ao SOA em educação.

- Uma plataforma para experimentação de diferentes SOA em estudantes.

- Desenvolvimento de um sistema de validação de SOA que leve em consideração o objetivo instrucional do educador para o sequenciamento instrucional.

A vantagem da utilização de modelos que possam descrever um SOA, como o ASSIMILATE, é que a representação é capaz de extensa análise por computadores. Apesar da informação que possa ser derivada de uma análise computadorizada não poder dizer que uma sequência de objetos de aprendizagem é boa ou ruim, a análise pode ajudar a localizar pontos onde essa sequência seja inconsistente, incompleta ou incorretamente especificada no planejamento e na execução instrucional. Do mesmo modo, é possível identificar pontos em que a sequência obtenha bons resultados conforme os objetivos instrucionais definidos pelos educadores. Localizando esses pontos no processo de planejamento e execução é possível que alguns problemas possam ser corrigidos; que o consumo de recursos como tempo e dinheiro sejam minimizados e a eficiência do processo instrucional seja melhorada. 


\section{Referências}

Brusilovsky, P. (2001). Developing Adaptive educational hypermedia. In International PEG Conference. . http://www.sis.pitt.edu/ p peterb/papers/PEG01.html, [accessed on Jun 8].

Brusilovsky, P. L. (1992). A framework for intelligent knowledge sequencing and task sequencing. In Intelligent tutoring systems. . Springer. http://link.springer.com/chapter/10.1007/3-540-55606-0_59, [accessed on Jun 20].

Brusilovsky, P. and Vassileva, J. (2003). Course sequencing techniques for large-scale web-based education. International Journal of Continuing Engineering Education and Life Long Learning, v. 13, n. 1, p. 75-94.

Chi, Y.-L. (2009). Ontology-based curriculum content sequencing system with semantic rules. Expert Systems with Applications, v. 36, n. 4, p. 7838-7847.

Gagne, R. M. (1962). The acquisition of knowledge. Psychological review, v. 69, n. 4, p. 355.

Gagne, R. M. (1963). Learning and proficiency in mathematics. The Mathematics Teacher, p. 620-626.

Gagne, R. M. (1968). Presidential address of division 15 learning hierarchies. Educational psychologist, v. 6, n. 1, p. 1-9.

Gagne, R. M. and Paradise, N. E. (1961). Abilities and learning sets in knowledge acquisition. Psychological Monographs: General and Applied, v. 75, n. 14, p. 1.

Gordon, J. L. (2000). Creating knowledge maps by exploiting dependent relationships. Knowledge-based systems, v. 13, n. 2, p. 71-79.

Karampiperis, P. and Sampson, D. G. (2004). Adaptive Instructional Planning Using Ontologies. In ICALT. . http://www.general.nsysu.edu.tw/gena/gena02/mlsp/karampontology-2004.pdf, [accessed on May 25].

Katuk, N. and Ryu, H. (2010). Finding an optimal learning path in dynamic curriculum sequencing with flow experience. In Computer Applications and Industrial Electronics (ICCAIE), 2010 International Conference on. . IEEE. http://ieeexplore.ieee.org/xpls/abs_all.jsp?arnumber=5735080, [accessed on Jul 2].

Kontopoulos, E., Vrakas, D., Kokkoras, F., Bassiliades, N. and Vlahavas, I. (2008). An ontology-based planning system for e-course generation. Expert Systems with Applications, v. 35, n. 1-2, p. 398-406.

Miller, P. L. and Phillips, E. R. (1974). Development of a learning hierarchy for the computacional Skills of rational number subtraction.

Murray, T. (1999). Authoring intelligent tutoring systems: An analysis of the state of the art. International Journal of Artificial Intelligence in Education (IJAIED), v. 10, p. $98-129$.

Murray, T. and Arroyo, I. (2002). Toward measuring and maintaining the zone of proximal development in adaptive instructional systems. In Intelligent Tutoring Systems. . Springer. http://link.springer.com/chapter/10.1007/3-540-47987-2_75, [accessed on Jun 27]. 
Nwana, H. S. (1990). Intelligent tutoring systems: an overview. Artificial Intelligence Review, v. 4, n. 4, p. 251-277.

Peachey, D. R. and McCalla, G. I. (1986). Using planning techniques in intelligent tutoring systems. International Journal of Man-Machine Studies, v. 24, n. 1, p. 7798.

Phillips, E. R. and Kane, R. B. (may 1973). Validating Learning Hierarchies for Sequencing Mathematical Tasks in Elementary School Mathematics. Journal for Research in Mathematics Education, v. 4, n. 3, p. 141.

Piaget, J. (1979). A construção do real na criança. Rio de Janeiro: Zahar.

Reigeluth, C. M. (1999). Instructional-Design Theories and Models. v. II

Resnick, L. B. (1975). Task Analysis in Instructional Design: Some Cases from Mathematics.

Sicilia, M.-Á., Lytras, M. D., Sánchez-Alonso, S., García-Barriocanal, E. and ZapataRos, M. (2011). Modeling instructional-design theories with ontologies: Using methods to check, generate and search learning designs. Computers in Human Behavior, v. 27, n. 4, p. 1389-1398.

Trembath, R. J. and White, R. T. (1975). Use of learning hierarchies in promoting mastery learning. Research in Science Education, v. 5, n. 1, p. 135-142.

Vidal-Castro, C., Sicilia, M.-Á. and Prieto, M. (2012). Representing instructional design methods using ontologies and rules. Knowledge-Based Systems, v. 33, p. 180-194.

Wiley, D. A. (2006). Connecting learning objects to instructional design theory: A definition, a metaphor, and a taxonomy.

Zuasnábar, D. M. H., Santibañez, M. R. F. and Fernandes, C. T. (1999). Ambiente de Apoio à Pré Autoria de Cursos Multimídia. . UFPR. 